\title{
IMPORTANTES CARACTERÍSTICAS DE CHUVA PARA A CONSERVAÇÃO DO SOLO E DA ÁGUA NO MUNICÍPIO DE SÃO MANUEL (SP) $)^{(1)}$
}

\author{
D. MORETI (2), M. P. CARVALHO(3), \\ A. R. MANNIGEL ${ }^{(2)} \&$ L. R. MEDEIROS ${ }^{(2)}$
}

\begin{abstract}
RESUMO
Os modelos matemáticos preditivos da erosão do solo, como a Equação Universal de Perda de Solo (E UPS), são de muita valia no planejamento de uso agrícola da terra. Tal equação, desenvolvida para estimar as perdas médias anuais de solo esperadas em dado local, para determinado sistema de manejo, apresenta como variáveis os fatores erosividade da chuva $(R)$, erodibilidade do solo (K), comprimento do declive (L), grau do declive (S), cobertura e manejo (C) e práticas conservacionistas de suporte $(P)$. Com o objetivo de contribuir para o planejamento conservacionista de uso do solo local, foi estimado, de forma simplificada, o fator erosi vidade da chuva (R) da EUPS para o município de São Manuel (SP), para uma série pluviométrica contínua de $\mathbf{4 9}$ anos de dados de chuva diária. Além disso, foram também calculados o período de retorno, a freqüência de ocorrência dos índices de erosividade anuais e as quantidades máximas diárias das chuvas necessárias para o dimensi onamento mais adequado de canais de terraços agrícolas em nível. $\mathbf{O}$ valor calculado do fator $\mathbf{R}$ foi de $7.487 \mathrm{MJ} \mathrm{mm} \mathrm{ha}^{-1} \mathrm{~h}^{-1}$ ano-1, esperado ocorrer no local, pelo menos, uma vez a cada 2,33 anos, com uma probabilidade de $42,92 \%$. Observou-se uma concentração de $81,48 \%$ do valor total deste fator no semestre de outubro a março, indicando que, potencialmente, as maiores perdas anuais de solo por erosão são esperadas neste período. Os valores anuais do índice $\mathrm{EI}_{30}$, esperados para os períodos de retorno de 2, 5, 10, 20, 50 e 100 anos, foram de 7.216, 8.675, 9.641, 10.568, 11.768 e

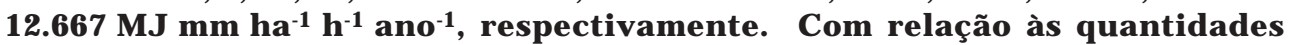
máximas de chuva diária, para os mesmos períodos de retorno, os valores foram de 73, 98, 115, 131, 151 e $167 \mathrm{~mm}$, respectivamente.
\end{abstract}

Termos de indexação: precipitação pluviométrica, erosividade, período de retorno da chuva.

\footnotetext{
(1) Trabal ho realizado na disciplina de Manejo e Conservação do Solo do Curso de Pós-Graduação em Agronomia, Área de Concentração em Sistema de Produção, Faculdade de Engenharia, Campus de Ilha Solteira - UNESP. Recebido para publicação em dezembro de 2001 e aprovado em abril de 2003.

(2) Mestrando em Manejo e Conservação do Solo, Faculdade de Engenharia, Campus de Il lha Solteira - UNESP. Caixa Postal 31, CEP 15.385-000 Il ha Solteira (SP). E-mail: dmoreti@carpa.ciagri.usp.br

(3) Professor Adjunto, Faculdade de Engenharia, UNESP. E-mail: morel @agr.feis.unesp.br
} 


\title{
SUMMARY: IMPORTANT RAINFALL CHARACTERISTICS FOR SOIL AND WATER CONSERVATION IN SÃO MANUEL, STATE OF SÃO PAULO, BRAZIL
}

\begin{abstract}
Mathematical models for soil erosion prediction are of unquestionable validity for planning the use of agricultural soils. The Universal Soil Loss Equation (USLE) is an exampl eof such model s and esti mates theexpected annual mean losses of soil in a gi ven site and for a given management system based on the parameters: rain erosivity (R), soil erodibility (K), slope length (L), steepness (S), soil cover and management $(C)$, and conservation practices $(\mathrm{P})$. With the objective of contributing to conservation planning in the use of local soils, the rain erosivity factor $(R)$ of theUSLE was estimated in si mplified form for a 49 year daily rain series in the county of São Manue, Brazil (22 45 ' S latitude; 48 30 ' W longitude). Besides, thereturn peri od, frequency of occurrence of theannual rain erosivity indexes, and themaximum daily rain values for thecalculation of canal dimension of agricultural level terraces werealso calculated. Thecalculated value of parameter $\mathrm{R}$ was $7,487 \mathrm{MJ} \mathrm{mm} \mathrm{ha-1} \mathrm{h}^{-1}$ year $^{-1}$, expected to occur at least onceevery 2.33 years at the site with a probability of $42.92 \%$. A concentration of $81.48 \%$ of the total $R$ value was observed from October through March, indicating that the greatest part of the annual erosion soil losses are expected to occur during this period. The values of the annual $\mathrm{EI}_{30}$ indexes for return periods of $2,5,10,20,50$, and 100 years, were 7,216; 8,675; 9,641; 10,568; 11,768 and $12,667 \mathrm{MJ} \mathrm{mm} \mathrm{ha-1} \mathrm{h}^{-1}$ year- ${ }^{-1}$, respectively. Maximum daily rain quantities were 73,98 , 115, 131, 151 and $167 \mathrm{~mm}$, respectively, for the same return periods.
\end{abstract}

Index terms: rain, erosivity, rain return period.

\section{NTRODUÇÃO}

A Equação Universal de Perda de Solo - EUPS (Wischmeier \& Smith, 1978) tem por objetivo predizer a erosão, em t ha-1ano-1, que poderá ocorrer em determinada área agrícola, de forma que a magnitude da perda desolo fiquesituada dentro dos limites toleráveis. Esta equação relaciona, de forma combinada, todos os fatores que influenciam a erosão acel erada do solo, quais sejam: erosividade da chuva $(\mathrm{R})$, erodibilidade do solo $(\mathrm{K})$, comprimento e grau do declive (LS), cobertura e manejo do solo (C) e práticas conservacionistas de suporte $(P)$. Dessa forma, sua utilização é de fundamental importância para o estabel ecimento do uso e manejo corretos do solo e, conseqüentemente, para a sel eção das práticas conservacionistas de maior eficácia na redução da erosão hídrica.

O solo é o recurso natural mais intensamente utilizado na produção de alimentos, podendo, por isso, ter sua capacidade produtiva comprometida pela erosão, em decorrência de seu uso e manejo inadequados. Assim, o conhecimento das relações entre os fatores que causam as perdas de solo e os que permitem reduzi-las é de fundamental importância para o planejamento conservacionista da propriedadeagrícola (Roque et al., 2001). Dentre os fatores, destaca-sea erosividadeda chuva (fator $\mathrm{R}$ da EUPS), que denota o potencial da chuva e enxurrada associada em causar erosão do sol o, sendo determinada pelo produto da energia cinética total da chuva com sua intensidade máxima em trinta minutos (Wischmeier \& Smith, 1978).

Fundamentalmente, a erosividade, definida como o potencial da chuva em causar erosão do solo, depende de suas características físicas básicas, a saber: tamanho, forma eveloci dadeterminal dequeda das gotas. Combinadamente, estas características determinam a energia cinética total da chuva.

Com o propósito de estudar este fator erosivo, a pesquisa tem demonstrado que as características da chuva que proporcionam as correlações mais el evadas com as perdas de sol o são a intensidadeea energia cinética. Já com relação à enxurrada, basicamente são o seu volume e taxa máxima de descarga as características mais importantes (Hudson, 1973; Lombardi Neto, 1977; Wischmeier \& Smith, 1978).

Founier(4), citado por Lombardi Neto (1977), verificou uma estreita correlação entreo que chamou de degradação específica ou total de erosão anual e o coeficiente de chuva c, expresso como:

$$
\mathrm{c}=\mathrm{p}^{2} / \mathrm{p}_{\mathrm{n}}
$$

sendo $\mathrm{p}$ a média mensal e $\mathrm{p}_{\mathrm{n}}$ a média anual dechuva. Com vistas em obter uma estimativa simplificada do índice $\mathrm{EI}_{30}$, uma vez que para muitos locais do

\footnotetext{
(4) FOURNIER, F. Climate et Erosion. Paris, Press Universitaires de France, Paris, 1960. 201p.
} 
estado de São Paulo não existem registros pluviográficos, Lombardi Neto (1977) estimou o índice de erosividade médio mensal no município de Campinas (SP) por meio de um coeficiente de chuva semelhante ao coeficiente da equação 1 , utilizando, exclusivamente, registros pluviométricos e a seguinte equação:

$$
\mathrm{R}_{\mathrm{c}}=\mathrm{P}_{\mathrm{m}}{ }^{2 / \mathrm{P}_{\mathrm{a}}}
$$

sendo $R_{c}$ o coeficiente de chuva, $P_{m}$ a precipitação pluviométrica média mensal e $\mathrm{P}_{\mathrm{a}}$ a precipitação pluviométrica média anual, em $\mathrm{mm}$, resultando na seguinte expressão $(r=0,991)$ :

$$
\mathrm{EI}_{30}=67,414 \mathrm{R}_{\mathrm{C}}{ }^{0,850}
$$

sendo $\mathrm{EI}_{30}$ a estimativa (por meio da equação de ajuste) do índice de erosividade médio mensal, em MJ $\mathrm{mm} \mathrm{ha}^{-1} \mathrm{~h}^{-1}$. Portanto, pode-se deduzir que, para uma localidade com uma distribuição de chuva semelhante à de Campinas, com uma série pluviométrica contínua de, no mínimo, 20 anos, tal equação pode estimar com boa aproximação os valores médios mensais do $\mathrm{EI}_{30}$, usando-se apenas dados de quantidade de chuva, isto é, dados pluviométricos. Posteriormente, inúmeras equações foram usadas com o mesmo objetivo daquela (equação 3) de Lombardi Neto (1977), tanto para outras localidades paulistas, como para o resto do País, de forma geral (Pereira, 1983; Carvalho, 1987; Sosa, 1987; Morais et al., 1991; Bertol, 1993, 1994; Colodro, 1999; Roque et al., 2001; Coelho, 2001).

O período de retorno, definido como o intervalo de tempo, em anos, dentro do qual determinado evento hidrológico máximo será igualado ou ultrapassado, é outro importante parâmetro de chuva. Ele pode ser determinado pelo método de Gumbel, que apresenta, como base estatística, a teoria dos valores extremos. Dessa forma, os val ores máxi mos anuais de determi nado evento aproximamse de um padrão definido de distribuição de freqüência quando o número de observações, em cada ano, torna-se o mais amplo possível (Schwab et al., 1966). No caso do terraceamento agrícola (Schwab et al., 1966; Lombardi Neto, 1989), tal evento hidrológicoécal culado normal mentepara um período de retorno de 10 a 15 anos.

Segundo Lombardi Neto (1989), noterraceamento em nível, o evento hidrológico máximo a ser utilizado érepresentado pela chuva máxima diária, enquanto, no terraceamento em desnível, é representado pela intensidade máxima de uma chuva com duração igual ao tempo de concentração da bacia. A escolha do período de retorno é necessária para a construção de obras hidráulicas de várias finalidades, incluindo as de grande porte, para garantir a segurança das populações ou áreas abaixo de onde elas serão construídas, uma vez que o princípio envolvido é o de eventos climatológi cos extremos (Schwab et al., 1966).
Por ser o período de retorno utilizado para o terraceamento agrícola normalmente de 10 a 15 anos, pode-se, eventual mente, planejar tal prática conservacionista do solo com uma margem de segurança maior, visando solucionar problemas específicos, considerando os de ordem econômica e ecológica e os possíveis danos maiores presumíveis, adotando períodos de retorno mais convenientes, mesmo que superestimem a dimensão normal dos canais dos terraços, como de 20 a 50 anos (Carvalho, 1987).

Dentro do enfoque tratado, o objetivo do presente trabalho foi estudar algumas características de chuva para o município de São Manuel (SP), relacionadas com erosividade da chuva.

\section{MATERIAL E MÉTODOS}

O trabal ho foi realizado a partir de dados obtidos em pluviômetro, registrados para o município de São Manuel (SP), fornecidos pela Coordenadoria de Assistência Técnica I ntegral local (CATI/Secretaria da Agricultura e Abastecimento do Estado de São Paulo). O município de São Manuel encontra-se próximo às coordenadas de $22^{\circ} 45^{\prime}$ de latitude sul e $48^{\circ} 30$ ' de longitude a oeste de Greenwich. Sua altitude média é de $520 \mathrm{~m}$, com as temperaturas médias mínimas e máximas, no verão, 19 e $29^{\circ} \mathrm{C}$ e, no inverno de 12 e $25^{\circ} \mathrm{C}$, respectivamente. O tipo climático predominantena regiãoéo Cwa, deacordo com o critério de Köeppen, caracterizado como clima tropical dealtitude, com inverno seco e verão quente (Nascimento \& Pereira, 1988).

Foram acumulados os dados diários deprecipitação pluviométrica da série compreendida entre 1950 e 1998 (49 anos). A precipitação mensal foi constituída do somatório das preci pitações diárias que ocorreram em determinado mês, dentro de um ano qualquer. Da mesma forma, a precipitação anual foi constituída do somatório de seus totais mensais, efetuando-se o mesmo para todos os anos da série. Assim, puderam ser obtidos os valores médios mensais da precipitação pluviométrica, mês a mês, para todos os meses do ano. Procedeu-se à tabulação de tais dados de forma a obter, finalmente, a precipitação média anual do município de São Manuel, para a referida série de chuva.

Para obter uma estimativa aproximada doíndice de erosividade mensal $\left(\mathrm{EI}_{30}\right)$, uma vez que para muitos locais do estado de São Paulo não existem dados pluviográficos, Lombardi Neto (1977) estimou o mesmo para o município de Campinas (SP) por meio do coeficiente de chuva relacionado na equação 2 , originado exclusivamente de dados pluviométricos (quantidade total de chuva). A correlação entre tal coeficiente e a precipitação média mensal resultou em elevado coeficiente de 
correlação para o modelo matemático do tipo potência, dado pela equação 3. Portanto, pode-se deduzir que, para uma localidade paulista que possua uma distribuição de chuva semel hante à de Campinas, com uma série pluviométrica contínua de, no mínimo, 20 anos, a equação 3 pode estimar com boa aproximação os valores médi os mensais do índice $\mathrm{EI}_{30}$, usandoapenas dados de quantidade total de chuva. Desta forma, tomando como base a metodologia de estimativa do índice $\mathrm{EI}_{30}$ por meio do coeficiente de chuva como o utilizado por Lombardi Neto (1977), os índices de erosividade médios mensais para o município de São Manuel (SP) foram obtidos por meio da seguinte expressão $(r=0,991)$, desenvolvida para o município de M ococa (SP) (Carvalho,1987), para o mesmo propósito como o da equação 3:

$$
\mathrm{EI}_{30}=111,173 \mathrm{Rc}^{0,691}
$$

em que $\mathrm{El}_{30}$ representa oíndice de erosividademédio mensal em $\mathrm{MJ} \mathrm{mm} \mathrm{ha}^{-1} \mathrm{~h}^{-1}$, obtido mês a mês, $\mathrm{e}_{c} \mathrm{O}$ coeficiente de chuva, obtido pelo quociente entre o quadrado da precipitação pluviométrica média mensal ea preci pitação pluviométrica média anual (equação 2). A validade de aplicação da equação obtida para Mococa aos dados de São Manuel foi previamente verificada, tanto por meio de regressão linear entre os dados de precipitação anual para a série de 1949 a 1998 das duas localidades, quanto por meio dos intervalos de confiança entre suas respectivas precipitações médias mensais, visando à extrapolação do uso da referida equação.

Os índices de erosividade, mês a mês, dentro de cada ano, foram estimados a partir do quadro geral de distribuição da precipitação pluviométrica para o município de São Manuel por meio de regra de três simples, da seguinte forma:

$$
\text { ei }=(P M \times I E) / P A
$$

em que ei é o valor do índice de erosividade $\mathrm{EI}_{30}$ de um mês i contido num anoj da série de dados, PM é o total precipitado num mês i contido num ano j da série de dados, IE é o valor do índice de erosividade $\mathrm{EI}_{30}$ médio mensal, no qual o mês i está contido na série de dados, ePA é o valor da preci pitação média mensal no qual o mês i está contidona série de dados.

A obtenção dos índices deerosividade mês a mês, dentro de cada ano, proporcionou o quadro final de índices de erosividade $\mathrm{EI}_{30}$ de São Manuel, para o período de 1949 a 1998. Para tanto, o somatório dos índices de erosividade mês a mês, dentro de cada ano, constituiu o índice de erosividade para determinado ano. Finalmente, ofator $\mathrm{R}$ das chuvas do município de São Manuel foi obtido pelo valor médio entre os 49 anos contidos na série estudada.

Foram analisados ainda a probabilidade de ocorrência e o correspondente período de retorno, tanto para os índices de erosividade $\mathrm{EI}_{30}$ anuais, como para as quantidades máximas de chuva diária, da série parcial estudada para o município de São Manuel, compreendida entre 1949 e 1998. Para tais análises, foram utilizadas as teorias de probabilidade logarítmica (Chow, 1951; 1954) e dos valores extremos (Gumbel, 1941; 1954), preconizadas por Schwab et al. (1966).

A estimativa do período de retorno ( $T$, em anos) foi calculada por meio da seguinte expressão:

$$
\mathrm{T}=(\mathrm{N}+1) / \mathrm{m}
$$

em que $\mathrm{N}$ representa o número de anos da extensão da série parcial de dados e m o número de ordem, quando os eventos climatológicos são relacionados em ordem decrescente de magnitude. Desta forma, a probabilidade em \% (Pr), equivalente ao período de retorno, foi calculada como:

$$
\operatorname{Pr}=100 / \mathrm{T}
$$

A estimativa do valor teórico do evento climático (Xc), para os índices de erosividade $\mathrm{EI}_{30}$ anuais e para as chuvas máximas diárias, foi efetuada com a seguinte expressão:

$$
X c=X(1+C v k)
$$

em que $X$ representa a média aritmética dos eventos climáticos, Cv é o coeficiente de variação ek é ofator freqüência do evento climático, estimado para determinado período de retorno ( $T$ ) e obtido com a expressão (Chow, 1954):

$$
\mathrm{k}=(-2,45 .(\lambda+\ln (\ln \mathrm{T}-\ln (\mathrm{T}-1)))) / \pi
$$

em que $\lambda$ é a constante de Euler $(0,577)$.

Foi utilizado papel de probabilidade logarítmica para plotar os valores observados dos eventos climáticos, assim como para traçar a respectiva curva de probabilidadelogarítmica, tanto dos índices de erosividade $\mathrm{EI}_{30}$ anuais, como das chuvas máximas diárias. As probabilidades de ocorrência de determinado evento climático e seu correspondente período deretornoforam relacionadas, respectivamente, nas abscissas (inferior e superior) do gráfico. $\mathrm{Na}$ ordenada, foram relacionadas as magnitudes dos eventos climáticos, sendo os índices de erosividade em unidades $M J \mathrm{~mm} \mathrm{ha}^{-1} \mathrm{~h}^{-1}$ ano-1 $^{-1}$ e a quantidade de chuva máxima diária em unidades de $\mathrm{mm}$.

A determinação da significância da extensão da série parcial estudada foi efetuada pela seguinte expressão (Schwab et al., 1966):

$$
Y=(4,30 \cdot t \cdot \log \theta)^{2}+6
$$

sendo Y o número aceitável de anos de registro da série parcial de eventos climáticos, t o valor estatístico de Student, a 10 \% de significância, com $(y-6)$ graus de liberdade, e $\theta$ é a relação entre as magnitudes do evento climático com período de retorno de 100 anos e aquele com período de retorno de 2 anos. 


\section{RESULTADOS E DISCUSSÃO}

O quadro 1 apresenta a distribuição mensal e anual da precipitação pluviométrica do município de São Manuel, representante da série de 49 anos de análise de dados (1950 a 1998) utilizada para o cálculo do fator R local. A preci pitação média anual observada foi de $1557,7 \mathrm{~mm}$, com desvio-padrão e erro-padrão da média de, respectivamente, 345,5 e $49,4 \mathrm{~mm}$. O coeficiente de variação foi de $22,18 \%$. A adequação do número de anos de registro da série parcial de dados de precipitação anual revel ou que, a $1 \%$ de probabilidade, o tempo mínimo aceitável, obtido pela equação 10, contida em Schwab et al. (1966), é de 10,5 anos.

Quadro 1. Distribuição mensal e anual da precipitação pluviométrica no município de São Manuel (SP), no período de 1950 a 1998

Precipitação pluviométrica

Ano

jan. fev. mar. abr. maio jun. jul. ago. set. out. nov. dez. Total

\begin{tabular}{|c|c|c|c|c|c|c|c|c|c|c|c|c|c|}
\hline 50 & 310 & 180 & 220 & 60 & 30 & 18 & 0 & 26 & 85 & 69 & 165 & 246 & 1.409 \\
\hline 51 & 404 & 280 & 100 & 66 & 52 & 11 & 5 & 0 & 87 & 142 & 100 & 220 & 1.467 \\
\hline 52 & 157 & 420 & 347 & 34 & 0 & 51 & 0 & 0 & 20 & 185 & 129 & 98 & 1.441 \\
\hline 53 & 221 & 181 & 193 & 23 & 20 & 19 & 45 & 0 & 85 & 132 & 281 & 228 & 1.428 \\
\hline 54 & 241 & 266 & 111 & 44 & 186 & 67 & 93 & 0 & 15 & 61 & 20 & 221 & 1.325 \\
\hline 55 & 161 & 173 & 137 & 201 & 42 & 52 & 22 & 65 & 4 & 99 & 108 & 218 & 1.282 \\
\hline 56 & 118 & 326 & 73 & 200 & 199 & 126 & 123 & 57 & 93 & 173 & 9 & 73 & 1.570 \\
\hline 57 & 288 & 144 & 129 & 99 & 48 & 42 & 174 & 81 & 164 & 119 & 43 & 167 & 1.498 \\
\hline 58 & 346 & 180 & 163 & 32 & 205 & 106 & 1 & 2 & 113 & 172 & 87 & 234 & 1.641 \\
\hline 59 & 287 & 117 & 255 & 65 & 54 & 13 & 27 & $5 \overline{5}$ & 11 & 107 & 120 & 153 & 1.264 \\
\hline 60 & 236 & 354 & 129 & 60 & 46 & 52 & 0 & 11 & 20 & 139 & 178 & 249 & 1.474 \\
\hline 61 & 141 & 211 & 148 & 122 & 86 & 0 & 3 & 10 & 23 & 126 & 100 & 216 & 1.186 \\
\hline 62 & 174 & 241 & 372 & 58 & 13 & 110 & 6 & 41 & 23 & 259 & 49 & 137 & 1.483 \\
\hline 63 & 232 & 114 & 149 & 10 & 46 & 59 & 11 & 58 & 145 & 201 & 57 & 88 & 1.170 \\
\hline 64 & 20 & 342 & 27 & 50 & 50 & 33 & 83 & 37 & $\begin{array}{r}140 \\
79\end{array}$ & 129 & 131 & 307 & 1.288 \\
\hline 65 & 433 & 392 & 149 & 97 & 109 & 38 & 0 & 13 & 106 & 184 & 191 & 252 & 1.964 \\
\hline 66 & 92 & 119 & 56 & 32 & 27 & 7 & 19 & 27 & 72 & 188 & 56 & 271 & 966 \\
\hline 67 & 332 & 263 & 56 & 4 & 0 & 108 & 22 & 0 & 85 & 235 & 124 & 183 & 1.412 \\
\hline 68 & 251 & 163 & 98 & 33 & 8 & 25 & 9 & 45 & 12 & 103 & 70 & 201 & 1.018 \\
\hline 69 & 156 & 106 & 96 & 41 & 35 & 53 & 22 & 16 & 111 & 163 & 433 & 121 & 1.353 \\
\hline 70 & 181 & 394 & 43 & 30 & 59 & 67 & 12 & 104 & 101 & 68 & 78 & 151 & 1.288 \\
\hline 71 & 164 & 94 & 187 & 23 & 52 & 109 & 70 & 40 & 78 & 88 & 36 & 91 & 1.032 \\
\hline 72 & 387 & 409 & 54 & 55 & 44 & 7 & 148 & 58 & 110 & 243 & 172 & 127 & 1.814 \\
\hline 73 & 132 & 128 & 171 & 110 & 84 & 79 & 52 & 38 & 58 & 113 & 42 & 170 & 1.177 \\
\hline 74 & 132 & 55 & 431 & 35 & 20 & 147 & 0 & 25 & 16 & 188 & 51 & 391 & 1.491 \\
\hline 75 & 128 & 334 & 71 & 77 & 16 & 2 & 59 & 0 & 38 & 120 & 149 & 331 & 1.325 \\
\hline 76 & 263 & 229 & 205 & 66 & 213 & 89 & 86 & 148 & 210 & 144 & 341 & 192 & 2.186 \\
\hline 77 & 311 & 122 & 176 & 136 & 21 & 72 & 50 & 8 & 107 & 129 & 117 & 435 & 1.684 \\
\hline 78 & 123 & 176 & 281 & 0 & 205 & 38 & 53 & 5 & 65 & 121 & 215 & 187 & 1.469 \\
\hline 79 & 93 & 111 & 104 & 30 & 67 & 0 & 37 & 110 & 140 & 117 & 97 & 158 & 1.064 \\
\hline 80 & 208 & 268 & 153 & 121 & 13 & 53 & 0 & 18 & 67 & 80 & 184 & 412 & 1.577 \\
\hline 81 & 323 & 66 & 68 & 91 & 31 & 59 & 10 & 24 & 23 & 249 & 169 & 267 & 1.380 \\
\hline 82 & 326 & 267 & 173 & 64 & 75 & 177 & 61 & 26 & 16 & 242 & 264 & 384 & 2.075 \\
\hline 83 & 390 & 147 & 143 & 130 & 304 & 264 & 34 & 0 & 275 & 166 & 155 & 289 & 2.297 \\
\hline 84 & 193 & 79 & 112 & 158 & 31 & 0 & 7 & 130 & 111 & 37 & 100 & 179 & 1.137 \\
\hline 85 & 112 & 196 & 197 & 201 & 71 & 22 & 13 & 23 & 105 & 28 & 125 & 84 & 1.177 \\
\hline 86 & 133 & 261 & 386 & 52 & 92 & 0 & 12 & 179 & 100 & 70 & 192 & 393 & 1.870 \\
\hline 87 & 337 & 258 & 67 & 57 & 229 & 234 & 24 & 17 & 110 & 97 & 264 & 146 & 1840 \\
\hline 88 & 359 & 263 & 138 & 101 & 115 & 44 & 0 & 0 & 40 & 270 & 138 & 225 & 1.693 \\
\hline 89 & 484 & 210 & 152 & 119 & 84 & 52 & 96 & 49 & 103 & 53 & 105 & 271 & 1.778 \\
\hline 90 & 329 & 260 & 351 & 37 & 80 & 26 & 107 & 96 & 108 & 208 & 119 & 150 & 1.871 \\
\hline 91 & 337 & 387 & 360 & 152 & 52 & 88 & 36 & 25 & 144 & 188 & 164 & 215 & 2.148 \\
\hline 92 & 104 & 319 & 333 & 177 & 134 & 5 & 16 & 24 & 158 & 256 & 161 & 123 & 1.810 \\
\hline 93 & 307 & 423 & 192 & 85 & 188 & 93 & 15 & 57 & 270 & 59 & 86 & 228 & 2.003 \\
\hline 94 & 289 & 196 & 150 & 102 & 98 & 34 & 31 & 0 & 2 & 110 & 225 & 401 & 1.638 \\
\hline 95 & 413 & 356 & 190 & 118 & 88 & 60 & 94 & 0 & 108 & 209 & 66 & 233 & 1.935 \\
\hline 96 & 209 & 136 & 257 & 104 & 63 & 29 & 4 & 51 & 176 & 256 & 148 & 301 & 1.734 \\
\hline 97 & 725 & 139 & 82 & 94 & 136 & 194 & 32 & 19 & 159 & 215 & 235 & 199 & 2.229 \\
\hline 98 & 134 & 514 & 224 & 85 & 155 & 25 & 26 & 72 & 140 & 188 & 36 & 366 & 1.965 \\
\hline Média & 249,5 & 232,0 & 172,6 & 80,4 & 83,2 & 62,4 & 37,8 & 38,6 & 91,7 & 149,0 & 136,4 & 224,1 & 1.557 \\
\hline
\end{tabular}


Desta forma, em relação à caracterização da chuva de regiões temperadas, este fato diferiu do relatado por Newman (1970), o qual recomendou a utilização de uma série parcial de dados climáticos com, pelo menos, 22 anos. Diferiu também dos relatos de Wischmeier \& Smith (1978), que indicaram o tempo mínimo de 20 anos de dados para a série contínua destinada ao cál culo do fator $\mathrm{R}$.

No entanto, em relação à caracterização da chuva de regiões tropicais, o resultado encontrado ficou de acordo com os de Lombardi Neto (1977), Carvalho (1987), Sosa (1987), Lima (1995), Carvalho et al. (1996, 1997), Colodro (1999), Roque \& Carval ho (1999), Coel ho (2001) e Roque et al. (2001), os quais relataram tempos menores do que 20-22 anos para a série pluviográfica contínua destinada ao cálculo do fator $\mathrm{R}$.
A figura 1 apresenta os intervalos de confiança deter minados para as preci pitações médias mensais dos municípios de São Manuel e Mococa, no período de 1950 a 1998. O objetivo foi verificar a eficácia de utilização da equação 4 de M ococa (SP) para estimar os índices de erosividade médios mensais de outra localidade, desde que a distribuição de chuva seja semel hante. Pode-seobservar quea referida equação é relativamente satisfatória para uso aos dados da preci pitação deSão Manuel (SP), uma vez que, para todos os meses do ano, com exceção de novembro e dezembro, percebeu-se, nas duas localidades, sobreposição dos interval os de confiança obti dos para as precipitações médias mensais. O coeficiente de correlação ( $r$ ) obti do da regressão linear com os dados médios anuais de precipitação de tais locais foi de $0,9163$ ( $p<0,00002)$, sendo, portanto, el evado.

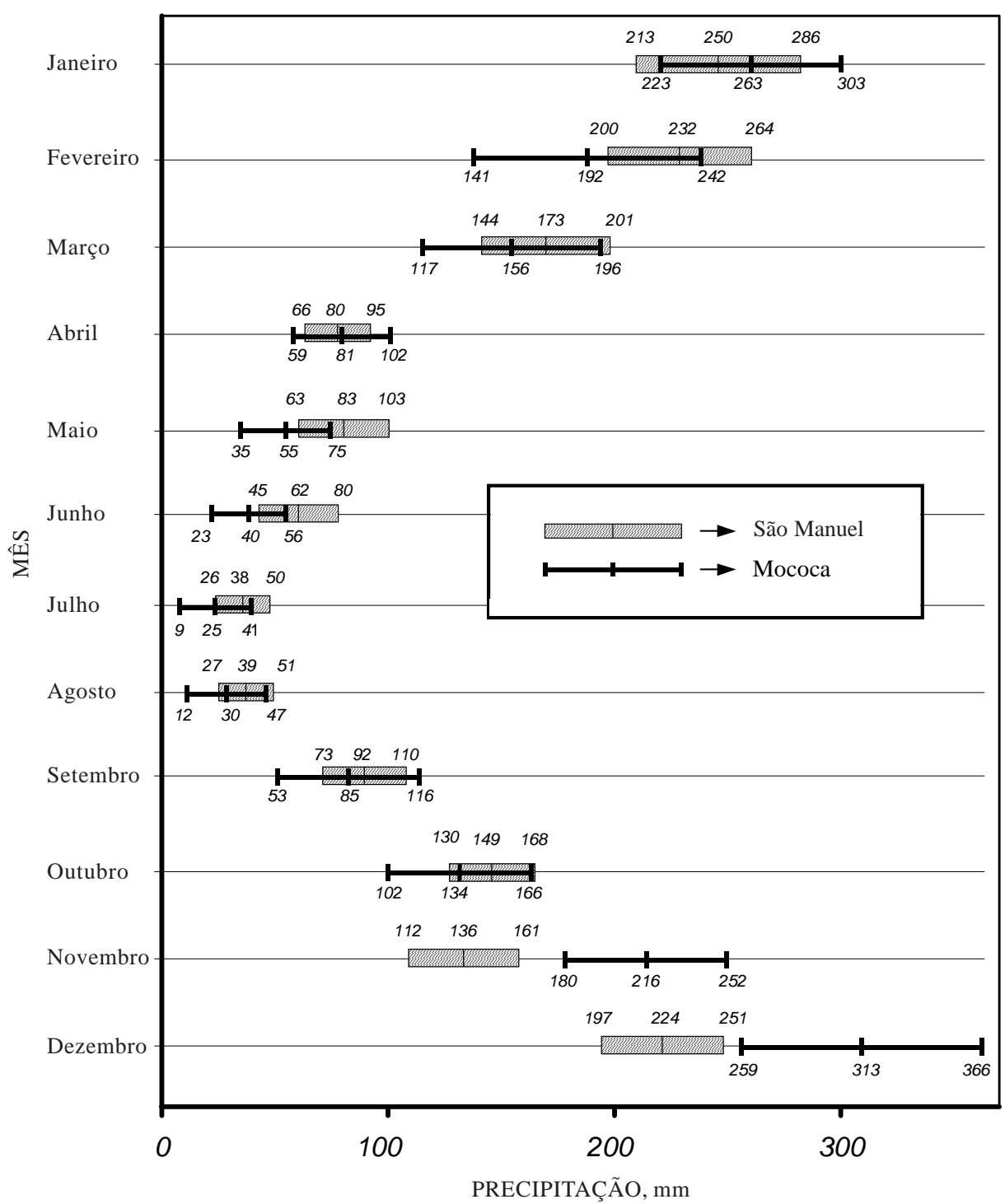

Figura 1. Intervalo de confiança da precipitação pluviométrica média mensal nos municípios de São Manuel (SP) e Mococa (SP), com base nas chuvas do período de 1950 a 1998. 
Os quadros 2 e 3 ea figura 2 apresentam os val ores médios mensais eanuais dofator erosividade da chuva (R) do município de São Manuel, avaliados para a série de dados entre os anos de 1950 a 1998. O fator $R$ local foi de $7.487 \mathrm{MJ} \mathrm{mm} \mathrm{ha-1} \mathrm{h}^{-1} \mathrm{ano}^{-1}$, com desviopadrão e erro-padrão da média de, respectivamente, 1.651 e $236 \mathrm{MJ} \mathrm{mm} \mathrm{ha}^{-1} \mathrm{~h}^{-1} \mathrm{ano}^{-1}$. O coeficiente de variação foi de $22,05 \%$.

O fator erosividade da chuva $(\mathrm{R})$ do município de SãoManuel de $7487 \mathrm{MJ} \mathrm{mm} \mathrm{ha}^{-1} \mathrm{~h}^{-1} \mathrm{ano}^{-1}$ (Quadros 2 e 3) foi superior aos de Mirandópolis, Piracicaba, Pindorama eSelvíria, calculados, respectivamente, por Kuratani (2001), Pereira (1983), Sosa (1987) e Carvalho et al. (1997). Por outro lado, foi inferior aos de Mococa, Barranquinho, Flexas e Bauru, calculados, respectivamente, por Carvalho (1987), Morais et al. (1991) e Lima (1995). Sua magnitude ficou um tanto em desacordo com o valor mostrado no mapa de linhas isoerosivas do estado de São Paulo, traçadas por Lombardi Neto et al . (1980), para o município de São Manuel, o qual está ao redor de $6.550 \mathrm{MJ} \mathrm{mm} \mathrm{ha-1} \mathrm{h}^{-1} \mathrm{ano}^{-1}$.

O estudo da distribuição do potencial erosivo da chuva, bem como da sua quantidade, é de grande importância para o manejo do sol o e das culturas. No período deestudo, os val ores anuais doíndice deerosividade variaram de 4.707 a $10.729 \mathrm{MJ} \mathrm{mm} \mathrm{ha-1} \mathrm{h}^{-1}$, respectivamente, para os anos de 1971 e 1997. Os valores mensais do índice de erosividade variaram de 105 e $1.766 \mathrm{MJ} \mathrm{mm} \mathrm{ha-1} \mathrm{h}^{-1}$, respectivamente, para os meses de julho e janeiro (Quadro 3). Analisando os dados apresentados, pode-se observar que $81,48 \%$ do índice de erosividade anual ocorreu nos meses de outubro a março, indicando que, neste período, era esperada a maior perda anual de solo por erosão no município de São Manuel (SP). Em contraposição, no semestre complementar (abril a setembro), esta quantidade foi de, apenas, 18,52 \% e, no trimestre de junho a agosto, ela foi de 5,63 \%. Tais valores, em termos de magnitude e época de ocorrência, concordam com os de pesquisas afins, realizadas em outras localidades do estado de São Paulo (Lombardi Neto, 1977; Sosa, 1987; Carvalho et al., 1989, 1996, 1997; Morais et al., 1991; Lima, 1995; Colodro, 1999, Roque et al., 2001 e Coel ho, 2001).

A distribuição da precipitação pluviométrica anual no município de São Manuel (Figura 1) seguiu um padrão semelhante ao unimodal, conforme descrição apresentada por Hudson (1973). Esta distribuição se assemelhou à dos municípios de Mococa, Campinas, Piraju e Votuporanga, respectivamente, determinadas por Carval ho (1987), Lombardi Neto (1977), Roque et al. (2001) e Coel ho (2001), que apresentaram um padrão de chuva com tendência ao tipo unimodal. No entanto, a distribuição foi diferente daquela do município de Teodoro Sampaio, determinada por Colodro (1999), a qual apresentou um padrão de chuva com tendência ao tipo bimodal.

A curva de distribuição da erosividade da chuva (Figura 2) fornece informações de grande importância para a determinação do fator cobertura emanejo (C) da Equação Universal dePerda de Solo e para a aplicação prática (Wischmeier \& Smith, 1965, 1978; Lombardi Neto, 1977; Carvalho, 1987). Os valores da distribuição relativa dos valores de $\mathrm{EI}_{30}$ médios mensais (estabel eci dos de jul ho a junho na figura 2 , de acordo com o ano agrícola), que poderão oportunamente ser utilizados no cál culo do fator $\mathrm{C}$ local, foram de, respectivamente, 1,40; 1,44; 4,$77 ; 9,31 ; 8,24 ; 16,38 ; 18,98 ; 17,16 ; 11,41 ; 3,97 ; 4,15$ e $2,79 \%$.

\section{Quadro 2. Valores médios mensais de chuva, coeficiente de chuva e índice de erosividade $\mathrm{EI}_{30}$ no município de São Manuel (SP), avaliados no período de 1950 a 1998}

\begin{tabular}{|c|c|c|c|}
\hline Mês & Chuva & $\begin{array}{c}\text { Coeficiente } \\
\text { de chuva }(\mathrm{Rc})^{(1)}\end{array}$ & $\mathrm{EI}_{30^{(2)}}$ \\
\hline & \multicolumn{2}{|c|}{$\longrightarrow \mathrm{mm}$} & MJ $\mathrm{mm} \mathrm{ha}^{-1} \mathrm{~h}^{-1} \mathrm{ano}^{-1}$ \\
\hline J aneiro & 249,5 & 39,96 & 1.421 \\
\hline Fevereiro & 232,0 & 34,55 & 1.285 \\
\hline Março & 172,6 & 19,13 & 854 \\
\hline Abril & 80,4 & 4,15 & 297 \\
\hline Maio & 83,2 & 4,44 & 311 \\
\hline J unho & 62,4 & 2,50 & 209 \\
\hline J ulho & 37,8 & 0,92 & 105 \\
\hline Agosto & 38,6 & 0,96 & 108 \\
\hline Setembro & 91,7 & 5,40 & 357 \\
\hline Outubro & 149,0 & 14,25 & 697 \\
\hline Novembro & 136,4 & 11,94 & 617 \\
\hline Dezembro & 224,1 & 32,24 & 1.226 \\
\hline Total & $1.557,7$ & -- & 7.487 \\
\hline
\end{tabular}

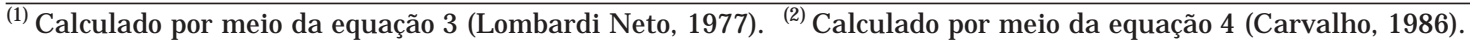


Quadro 3. Valores médios mensais e anuais do índice de erosi vidade $\mathrm{EI}_{30}$ no município de São Manuel (SP), com base nas chuvas do período de 1950 a 1998

\begin{tabular}{|c|c|c|c|c|c|c|c|c|c|c|c|c|c|}
\hline \multirow{2}{*}{ Ano } & \multicolumn{13}{|c|}{ Precipitação pluviométrica } \\
\hline & jan. & fev. & mar. & abr. & maio & jun. & jul. & ago. & set. & out. & nov. & dez. & Total \\
\hline & \multicolumn{13}{|c|}{ - MJ $\mathrm{mm} \mathrm{ha}^{-1} \mathrm{~h}^{-1} \mathrm{ano}^{-1}$} \\
\hline 50 & 1.766 & 997 & 1.089 & 222 & 112 & 60 & 0 & 73 & 331 & 323 & 746 & 1.346 & 7.064 \\
\hline 51 & 2.301 & 1.551 & 495 & 244 & 194 & 37 & 14 & 0 & 339 & 664 & 452 & 1.204 & 7.494 \\
\hline 52 & 894 & 2.326 & 1.717 & 126 & 0 & 171 & 0 & 0 & 78 & 865 & 584 & 536 & 7.297 \\
\hline 53 & 1.259 & 1.003 & 955 & 85 & 75 & 64 & 125 & 0 & 331 & 617 & 1.271 & 1.247 & 7.031 \\
\hline 54 & 1.373 & 1.473 & 549 & 163 & 695 & 224 & 258 & 0 & 58 & 285 & 90 & 1.209 & 6.379 \\
\hline 55 & 917 & 958 & 678 & 743 & 157 & 174 & 61 & 182 & 16 & 463 & 489 & 1.193 & 6.030 \\
\hline 56 & 672 & 1.806 & 361 & 739 & 744 & 422 & 342 & 159 & 362 & 809 & 41 & 399 & 6.856 \\
\hline 57 & 1.640 & 798 & 638 & 366 & 179 & 141 & 483 & 227 & 638 & 557 & 195 & 914 & 6.775 \\
\hline 58 & 1.971 & 997 & 807 & 118 & 766 & 355 & 3 & 6 & 440 & 805 & 394 & 1.280 & 7.940 \\
\hline 59 & 1.635 & 648 & 1.262 & 240 & 202 & 44 & 75 & 154 & 43 & 501 & 543 & 837 & 6.182 \\
\hline 60 & 1.344 & 1.961 & 638 & 222 & 172 & 174 & 0 & 31 & 78 & 650 & 805 & 1.362 & 7.437 \\
\hline 61 & 803 & 1.169 & 732 & 451 & 321 & 0 & 8 & 28 & 90 & 589 & 452 & 1.182 & 5.825 \\
\hline 62 & 991 & 1.335 & 1.841 & 214 & 49 & 368 & 17 & 115 & 90 & 1.212 & 222 & 749 & 7.201 \\
\hline 63 & 1.321 & 631 & 737 & 37 & 172 & 198 & 31 & 162 & 565 & 940 & 258 & 481 & 5.533 \\
\hline 64 & 114 & 1.894 & 134 & 185 & 187 & 111 & 231 & 104 & 308 & 603 & 593 & 1.680 & 6.141 \\
\hline 65 & 2.466 & 2.171 & 737 & 358 & 407 & 127 & 0 & 36 & 413 & 861 & 864 & 1.379 & 9.820 \\
\hline 66 & 524 & 659 & 277 & 118 & 101 & 23 & 53 & 76 & 280 & 879 & 253 & 1.483 & 4.727 \\
\hline 67 & 1.891 & 1.457 & 277 & 15 & 0 & 362 & 61 & 0 & 331 & 1.099 & 561 & 1.001 & 7.055 \\
\hline 68 & 1.430 & 903 & 485 & 122 & 30 & 84 & 25 & 126 & 47 & 482 & 317 & 1.100 & 5.149 \\
\hline 69 & 888 & 587 & 475 & 151 & 131 & 178 & 61 & 45 & 432 & 762 & 1.959 & 662 & 6.332 \\
\hline 70 & 1.031 & 2.182 & 213 & 111 & 221 & 224 & 33 & 291 & 393 & 318 & 353 & 826 & 6.196 \\
\hline 71 & 934 & 521 & 925 & 85 & 194 & 365 & 194 & 112 & 304 & 412 & 163 & 498 & 4.707 \\
\hline 72 & 2.204 & 2.265 & 267 & 203 & 164 & 23 & 411 & 162 & 428 & 1.137 & 778 & 695 & 8.739 \\
\hline 73 & 752 & 709 & 846 & 406 & 314 & 265 & 144 & 106 & 226 & 529 & 190 & 930 & 5.417 \\
\hline 74 & 752 & 305 & 2.133 & 129 & 75 & 492 & 0 & 70 & 62 & 879 & 231 & 2.139 & 7.267 \\
\hline 75 & 729 & 1.850 & 351 & 284 & 60 & 7 & 164 & 0 & 148 & 561 & 674 & 1.811 & 6.639 \\
\hline 76 & 1.498 & 1.268 & 1.014 & 244 & 796 & 298 & 239 & 414 & 818 & 674 & 1.543 & 1.050 & 9.856 \\
\hline 77 & 1.771 & 676 & 871 & 502 & 78 & 241 & 139 & 22 & 417 & 603 & 529 & 2.380 & 8.230 \\
\hline 78 & 701 & 975 & 1.390 & 0 & 766 & 127 & 147 & 14 & 253 & 566 & 973 & 1.023 & 6.935 \\
\hline 79 & 530 & 615 & 515 & 111 & 250 & 0 & 103 & 308 & 545 & 547 & 439 & 864 & 4.826 \\
\hline 80 & 1.185 & 1.484 & 757 & 447 & 49 & 178 & 0 & 50 & 261 & 374 & 832 & 2.254 & 7.871 \\
\hline 81 & 1.840 & 366 & 336 & 336 & 116 & 198 & 28 & 67 & 90 & 1.165 & 764 & 1.461 & 6.766 \\
\hline 82 & 1.857 & 1.479 & 856 & 236 & 280 & 593 & 169 & 73 & 62 & 1.132 & 1.194 & 2.101 & 10.033 \\
\hline 83 & 2.221 & 814 & 708 & 480 & 1.136 & 884 & 94 & 0 & 1.071 & 777 & 701 & 1.581 & 10.468 \\
\hline 84 & 1.099 & 438 & 554 & 584 & 116 & 0 & 19 & 364 & 432 & 173 & 452 & 979 & 5.210 \\
\hline 85 & 638 & 1.086 & 975 & 743 & 265 & 74 & 36 & 64 & 409 & 131 & 565 & 460 & 5.445 \\
\hline 86 & 757 & 1.446 & 1.910 & 192 & 344 & 0 & 33 & 501 & 389 & 327 & 869 & 2.150 & 8.918 \\
\hline 87 & 1.919 & 1.429 & 332 & 211 & 856 & 784 & 67 & 48 & 428 & 454 & 1.194 & 799 & 8.519 \\
\hline 88 & 2.045 & 1.457 & 683 & 373 & 430 & 147 & 0 & 0 & 156 & 1.263 & 624 & 1.231 & 8.408 \\
\hline 89 & 2.757 & 1.163 & 752 & 440 & 314 & 174 & 267 & 137 & 401 & 248 & 475 & 1.483 & 8.610 \\
\hline 90 & 1.874 & 1.440 & 1.737 & 137 & 299 & 87 & 297 & 269 & 420 & 973 & 538 & 821 & 8.892 \\
\hline 91 & 1.919 & 2.144 & 1.781 & 561 & 194 & 295 & 100 & 70 & 561 & 879 & 742 & 1.176 & 10.423 \\
\hline 92 & 592 & 1.767 & 1.648 & 654 & 501 & 17 & 44 & 67 & 615 & 1.198 & 728 & 673 & 8.504 \\
\hline 93 & 1.748 & 2.343 & 950 & 314 & 703 & 311 & 42 & 159 & 1.051 & 276 & 389 & 1.247 & 9.534 \\
\hline 94 & 1.646 & 1.086 & 742 & 377 & 366 & 114 & 86 & 0 & 8 & 515 & 1.018 & 2.194 & 8.151 \\
\hline 95 & 2.352 & 1.972 & 940 & 436 & 329 & 201 & 261 & 0 & 420 & 978 & 299 & 1.275 & 9.462 \\
\hline 96 & 1.190 & 753 & 1.272 & 384 & 235 & 97 & 11 & 143 & 685 & 1.198 & 669 & 1.647 & 8.285 \\
\hline 97 & 4.129 & 770 & 406 & 347 & 508 & 650 & 89 & 53 & 619 & 1.006 & 1.063 & 1.089 & 10.729 \\
\hline 98 & 763 & 2.847 & 1.108 & 314 & 579 & 84 & 72 & 201 & 545 & 879 & 163 & 2.002 & 9.559 \\
\hline Média & 1.421 & 1.285 & 854 & 297 & 311 & 209 & 105 & 108 & 357 & 697 & 617 & 1.226 & 7.487 \\
\hline
\end{tabular}

No quadro 4, são apresentados a probabilidade de ocorrência e o período de retorno dos valores dos índices anuais deerosividadee das chuvas máximas diárias nomunicípio deSãoManuel, duranteo período de 1950 a 1998. Observa-se que os val ores do período de retorno e da probabilidade de ocorrência, determinados para o maior valor doíndice de erosividade anual observado (10.729 $\mathrm{MJ} \mathrm{mm} \mathrm{ha}^{-1} \mathrm{~h}^{-1}$ ano $^{-1}$, 


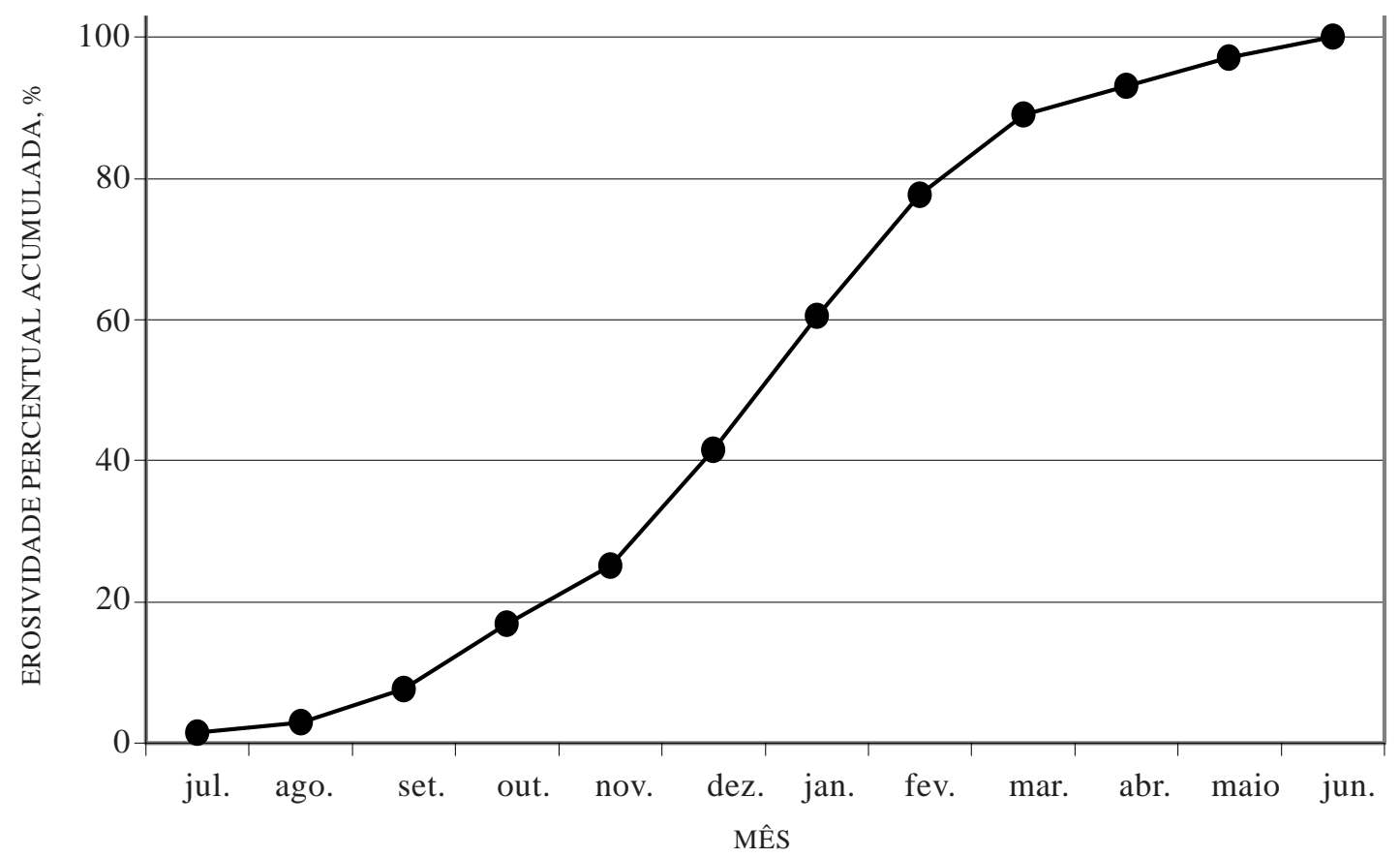

Figura 2. Distribuição relativa acumulada anual do índice de erosividade (EI30) no município de São Manuel (SP), com base nas chuvas do período de 1950 a 1998.

assim como para o maior valor de chuva máxima diária (160 mm), foram de, respectivamente, 50 anos e $2 \%$. Da mesma forma, os valores menores para o menor índice de erosividade anual echuva máxima diária (4.707 MJ mm ha-1 $\mathrm{h}^{-1} \mathrm{ano}^{-1}$ e $31 \mathrm{~mm}$ ) foram de, respectivamente, 1 ano e $98 \%$.

A distribuição da probabilidade de ocorrência, apresentada nas figuras 3 e 4, foi ajustada por meio da equação (8), de acordo com indicação de Schwab et al. (1966). Os valores dos índices anuais de erosividade e das chuvas máximas diárias, para qualquer período de retorno desejado, ou vice-versa, podem ser facilmente obtidos por meio de leitura direta nas curvas de distribuição de probabilidade apresentadas nas figuras 3 e 4, ou calculados por meio da aplicação da equação 8. Como exemplo, observa-se que o período de retorno do fator $\mathrm{R}$ (erosividade da chuva) do município de São Manuel é de 2,33 anos, para uma probabilidade de ocorrência de $42,92 \%$. Assim, pode-se dizer que, para o local estudado, é possível ocorrer, com uma probabilidade de $42,92 \%$, um valor anual do índice $\mathrm{El}_{30}$ de igual ou superior ao calculado (7.487 MJ mm ha-1 $\mathrm{h}^{-1}$ ano-1), pelo menos, uma vez a cada 2,33 anos. Os valores dos índices anuais de erosividade, esperados para os períodos de retorno de 2, 5, 10, 20, 50 e 100 anos, são, respectivamente, 7.216, 8.675, 9.641, 10.568, 11.768 e $12.667 \mathrm{MJ} \mathrm{mm} \mathrm{ha}^{-1} \mathrm{~h}^{-1} \mathrm{ano}^{-1}$.

As curvas de distribuição de probabilidade dos valores dos índices anuais de erosividade e das chuvas máximas diárias do município de São Manuel (SP) (Figuras 3 e 4) seguiram o padrão lognormal, típico de eventos hidrológicos, de acordo com Wischmeier (1959), e corroborados por Lombardi Neto (1977), Carvalho (1987), Sosa (1987), Lima (1995), Carval ho et al . (1996, 1997), Colodro (1999), Roque et al. (2001) e Coel ho (2001). Os val ores dos índices anuais de er osividadee das chuvas máximas diárias do município deSão Manuel, esperados para os períodos de retorno de 2, 5, 10, 20, 50 e 100 anos, no geral, diferiram daqueles de outras localidades do estado deSão Paulo, para as quais também foram realizados estudos semel hantes.

De acordo com os resultados constantes no quadro 4, omaior val or doíndice anual deerosividade (10.729 MJ mm ha-1 $\mathrm{h}^{-1} \mathrm{ano}^{-1}$ ) e o da quantidade de chuva máxima diária (160 mm), observados para São Manuel, apresentaram um período de retorno de 50 anos, equivalente a uma probabilidade de ocorrência de $2 \%$. Portanto, é esperado ocorrer neste local, com uma probabilidade de $2 \%$, um valor do índice anual deerosividade, ou valor da quantidade de chuva máxima diária, igual ou superior, respectivamente, a $10.729 \mathrm{MJ} \mathrm{mm} \mathrm{ha-1} \mathrm{h}^{-1}$ ano $^{-1} \mathrm{e}$ $160 \mathrm{~mm}$, pelo menos, uma vez a cada 50 anos.

Deforma semel hante, para o menor índice anual médio de erosividadee a menor quantidade de chuva máxima diária, espera-sequeocorra, com uma probabilidadede $98 \%$, umvalor doíndice anual deerosividade, ou da chuva máxima diária, igual ou superior, respectivamente, a $4.707 \mathrm{MJ} \mathrm{mm} \mathrm{ha}^{-1} \mathrm{~h}^{-1} \mathrm{ano}^{-1} \mathrm{e}$ $31 \mathrm{~mm}$, pelo menos, uma vez a cada 1 ano.

Os valores médios das chuvas máximas diárias, esperados para os períodos de retorno de 2, 5, 10, 20, 
Quadro 4. Período de retorno e probabilidade de ocorrência do índice $\mathrm{EI}_{30}$ anual e da quantidade de chuva máxima diária no município de São Manuel (SP), com base nas chuvas do período de 1950 a 1998

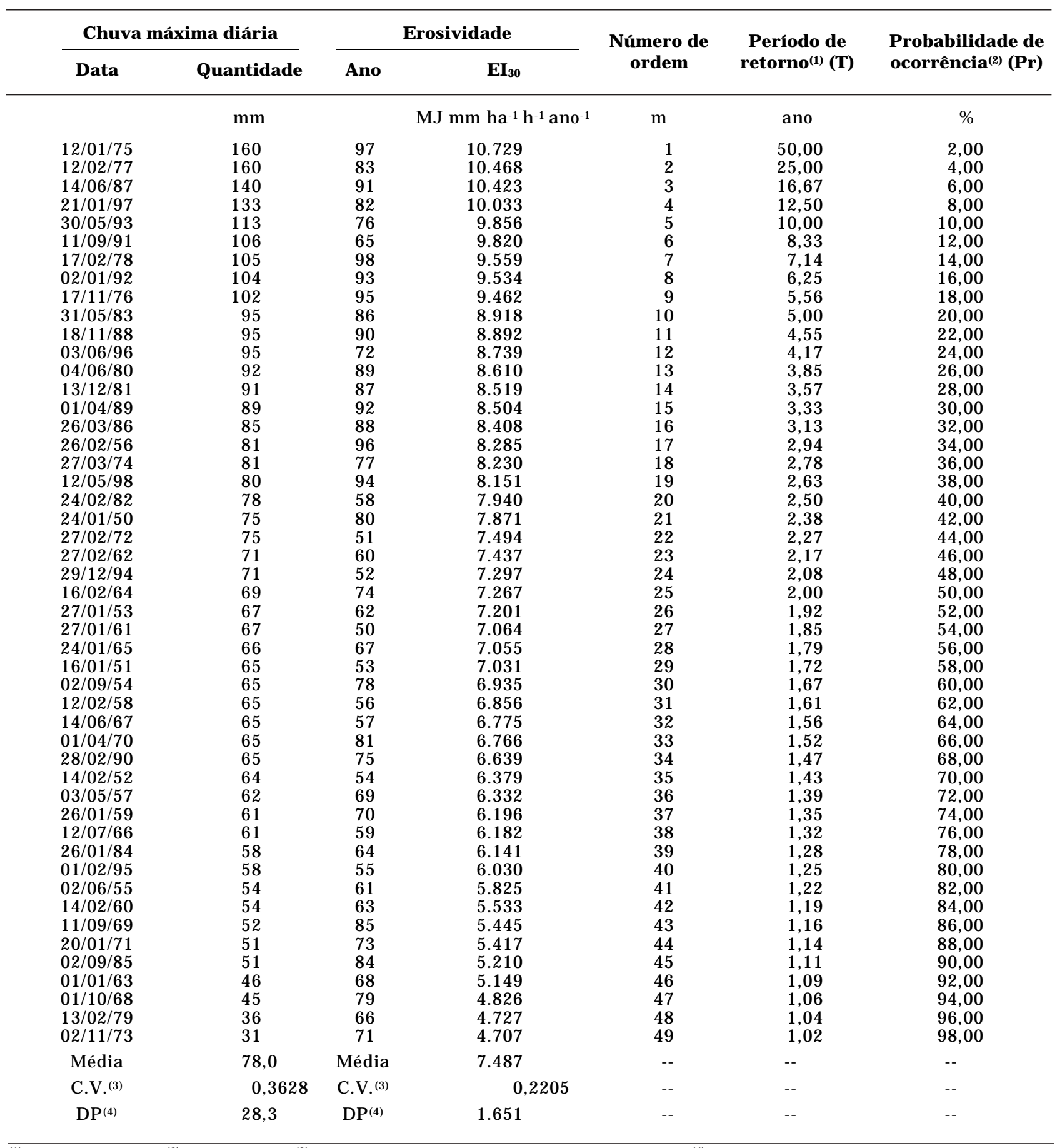

$\overline{(1)} \mathrm{T}=(\mathrm{N}+1) / \mathrm{m} \ldots . .{ }^{(2)} \mathrm{Pr}=100 / \mathrm{T} \ldots{ }^{(3)} \mathrm{CV}=$ Coeficiente de Variação (valor absoluto). ${ }^{(4)} \mathrm{DP}=$ Desvio-padrão.

50 e 100 anos, foram, respectivamente, de 73,4; 98,4; 115,$0 ; 130,8 ; 151,4$ e166,9 mm. O val or médio da chuva máxima diária (115 mm) para o período de retorno de 10 anos, utilizado no cálculo de área da seção transversal deterraços agrícolas em nível, conforme preceitos de Schwab et al. (1966) e Lombardi Neto (1989), concorda plenamente com o valor mostrado no mapa de isoi etas previamente determinado para o estado de São Paulo, uma vez que o município de São Manuel situa-se na isoi eta de 115,0 mm. 


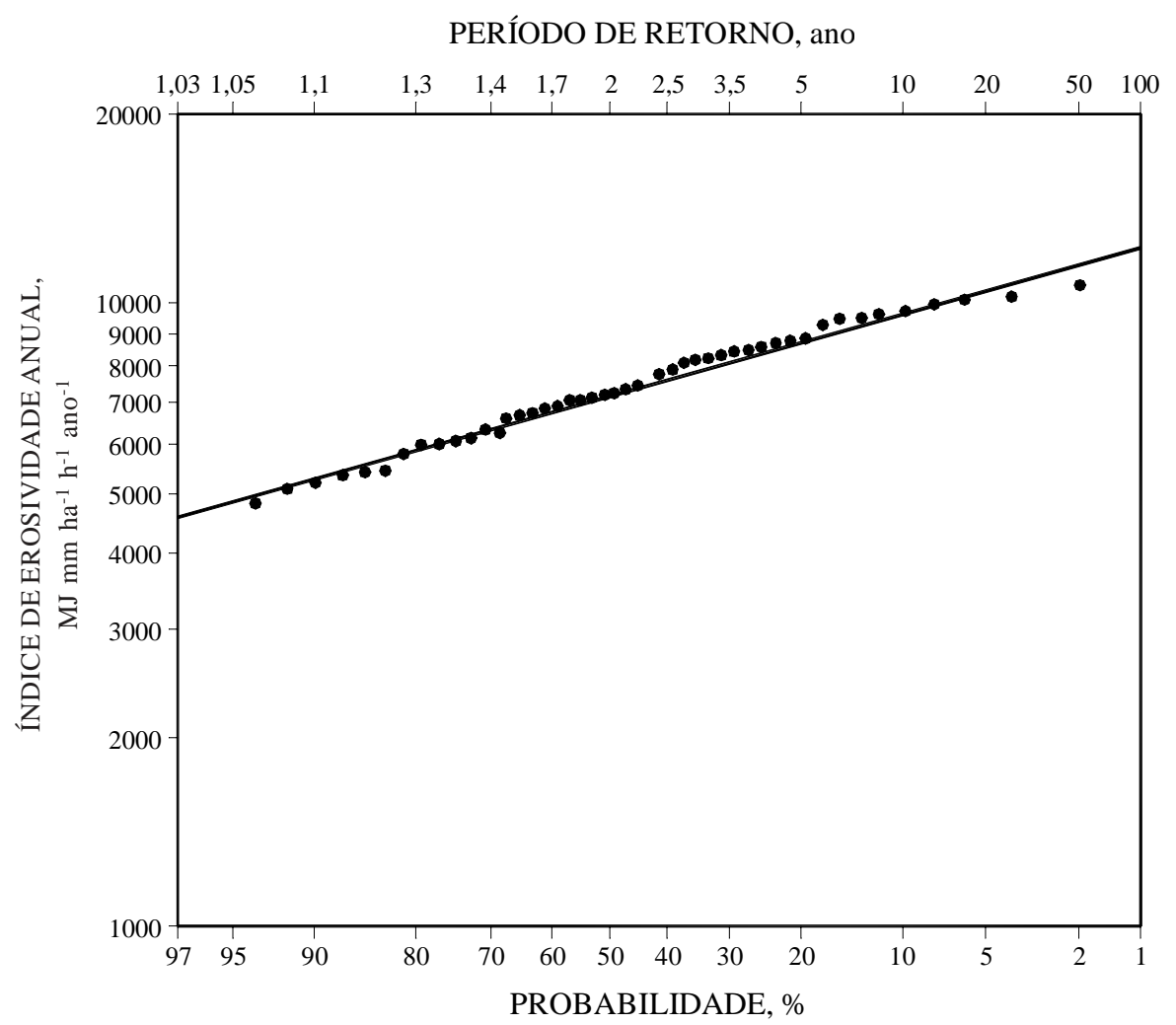

Figura 3. Distribuição da probabilidade de ocorrência e do período de retorno do índice EI 30 anual no município de São Manuel (SP), com base nas chuvas do período de 1950 a 1998.

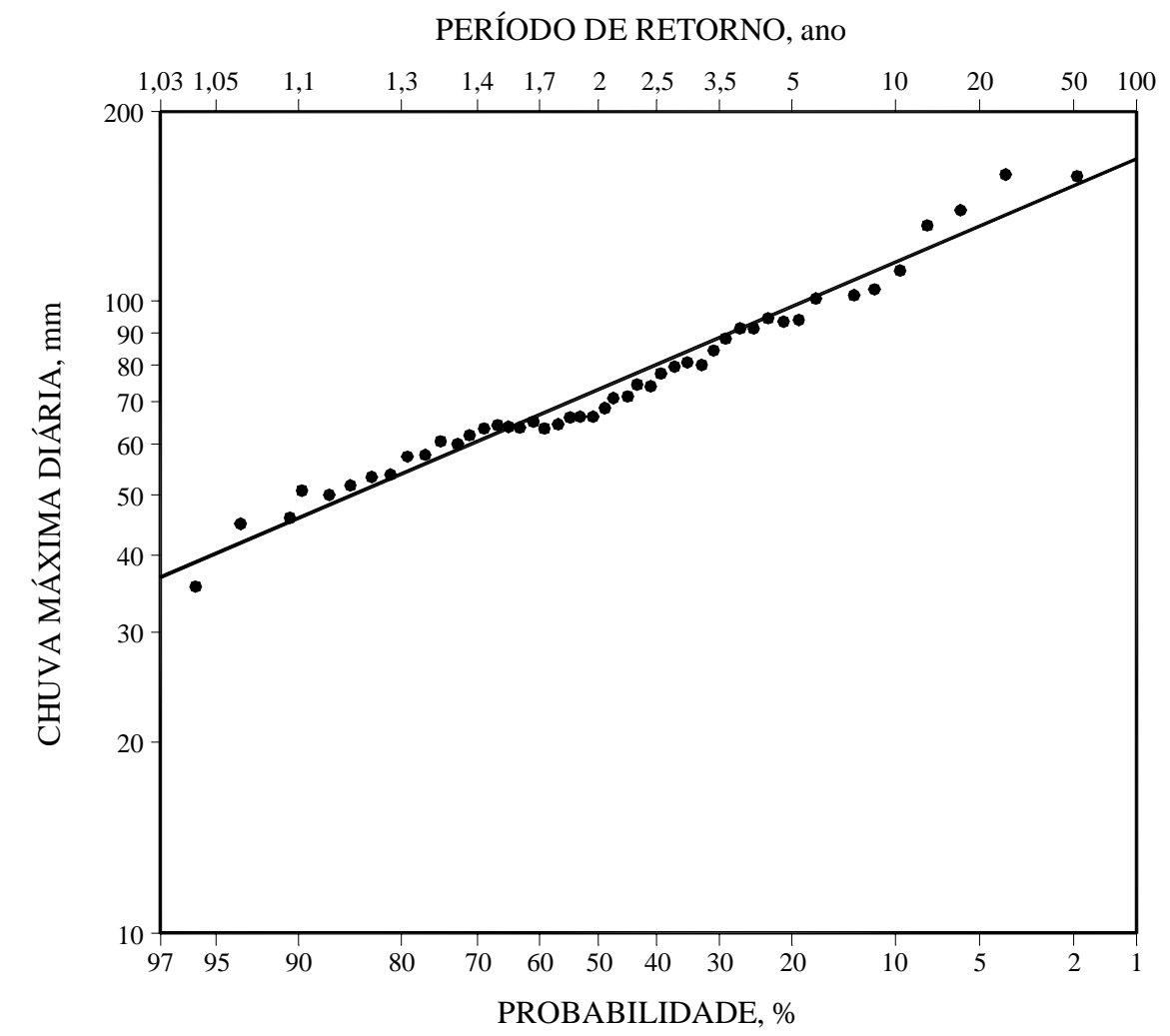

Figura 4. Distrı buıçao da probabıı aade de ocorrencıa e do perı odo de retorno da quantidade de chuva máxima diária no município de São Manuel (SP), com base nas chuvas do período de 1950 a 1998. 


\section{CONCLUSÕES}

1. Os dados referentes ao fator R de São Manuel do presentetrabal ho devem ser aceitos com um nível de significância de 99,10 \% de probabilidade de acerto. I sto porque, a equação de Mococa, que estimou a erosicidade da chuva de São Manuel, apresentou um coeficiente de correlação de 0,991.

2. A chuva máxima diária, calculada para o período de retorno de 10 anos e que é necessário ao terraceamento agrícola, foi de $115 \mathrm{~mm}$. Entretanto, desejando-se trabal har com maior segurança de tal prática conservacionista, podem ser utilizados valores mais consistentes, com períodos de retorno de 20, 50 e 100 anos, respectivamente de 130,8, 151,4 e $166,9 \mathrm{~mm}$.

\section{LITE RATURA CITADA}

BERTOL, I. Índice de erosividade $\left(\mathrm{EI}_{30}\right)$ para Lajes (SC). Ia aproximação. Pesq. Agropec. Bras., 28:515-521, 1993.

BERTOL, I. Avaliação da erosividade da chuva na localidade de Campos Novos (SC), no período de 1981 a 1990. Pesq. Agropec. Bras., 29:1453-1458, 1994.

CARVALHO, M.P. Erosividade da chuva: distribuição e correlação com as perdas de sol o de M ococa (SP). Piracicaba, Escola Superior de Agricultura "Luiz de Queiroz", 1987. 104p. (Tese de Mestrado)

CARVALHO, M.P.; LOMBARDI NETO, F.; VASQUES FILHO, J. \& CATANEO, A. Índices de erosividade da chuva correlacionados com as perdas de um Podzólico Vermel hoAmarelo eutrófico textura argilosa/muito argilosa de Mococa (SP): primeira aproximação do fator erodibilidade dos solos. R. Bras. Ci. Solo, 13:237-242, 1989.

CARVALHO, M.P.; LOMBARDI NETO, F.; VASQUES FILHO, J . \& CATANEO, A. Correlação entreoíndice de erosividade $\mathrm{EI}_{30}$ médio mensal e coeficiente de chuva do município de Mococa - SP. Científica, 19:1-7, 1991.

CARVALHO, M.P.; PISSARRA, T.C.T. \& AULINO, H.B. Erosividade da chuva de Botucatu (SP): distribuição, período de retorno e probabilidade de ocorrência. In: CONGRESSO LATINO AMERICANO DE CONSERVAÇÃO DO SOLO, 13., Águas de Lindóia, 1996, Águas de Lindóia. Anais. Piracicaba, Universidade de São Paulo, 1996. CD-ROM

CARVALHO, M.P.; PISSARRA, T.C.T. \& PAULINO, H.B. E rosividade da chuva de Sel viria (MS): distribuição, período de retorno e probabilidade de ocorrência. In: CONGRESSO BRASILEIRO DE CIÊNCIA DO SOLO, 26., Rio deJ aneiro, 1997. Anais. Campinas, Sociedade Brasileira de Ciência do Solo, 1997. CD-ROM

CHOW, V.T. General fórmula for hidrologic frequency analysis, Am. Geophys. Union Trans, 32:231-237, 1951.
CHOW, V.T. The log-probability law and II engineering applications, Americ Society. And Engeenerig, 1954. 80p. (Separate, 536)

COELHO, A.P. Fator erosividade da chuva de Votuporanga (SP). I Iha Solteira, Universidade Estadual Paulista, 2001. 101p. (Tese de Mestrado)

COLODRO, G. Erosividade da chuva: distribuição e correlação com a preci pitação pluviométrica de TeodoroSampaio (SP), I Iha Solteira, Universidade Estadual Paulista, 1999. 77p. (Tese de Mestrado)

GUMBEL, E.J. The return period of flood flows. Annals of mathematical statistics, 1941, v.12, n.2, june.

GUMBEL, E.J . Statistical theory of extreme values and some pratical applications. Bureau Standards, 1954. (Apllied Mathematicals Series, 33)

HUDSON, N.W. Soil conservation. 2.ed. Ithaca, Cornell University Press, 1973. 320p.

KURATANI, M.C.B. Erosividade da chuva de Mirandópol is (SP): Distribuição, período de retorno e probabilidade de ocorrência. Três Lagoas, Universidade Federal de Mato Grosso do Sul, 2001.55p. (Lato Sensu em Geografia)

LIMA, M.A. Avaliação do período de retorno e da probabilidade de ocorrência da erosividade da chuva de Bauru (SP)., I lha Solteira, Universidade Estadual Paulista, 1995.35p. (Trabalho de Graduação)

LOMBARDI NETO, F. Rainfall erosivity - its distribution and relationship with soil loss as Campinas, Brasil. West Lafayette, PurdueU niversity, 1977. 53p.(Tese de Mestrado)

LOMBARDI NETO, F.; SILVA, I.R.L. \& CASTRO, O.M. Potencial de erosão de chuvas no estado de São Paulo. In: ENCONTRO NACIONAL DE PESQUISA SOBRE CONSERVAÇÃO DO SOLO, 3., Recife 1980. Anais. Recife, Sociedade Brasileira de Ciência do Solo, 1980. p.159.

LOMBARDI NETO, F. Dimensionamento de terraço. In: SIMPÓSIO SOBRE TERRACEAMENTO AGRÍCOLA, 1988, Campinas. Simpósio. Campinas, Fundação Cargill, 1989. p.26-59.

MORAIS, L.F.B.; SILVA, V.; NASCHENVENG, T.M.; HARDOIN, P.C.; ALMEIDA, J .E.L.; WEBER, O.L.; BOEL, E. \&. DURIGON, V. Índice $\mathrm{EI}_{30}$ e sua correlação com o coeficiente de chuva do sudoeste do Mato Grosso. R. Bras. Ci. Solo, 15:339-344, 1991.

NASCIMENTO, C.M. \& PEREIRA, M.A.M.G. Atlas climatológico do estado de São Paulo. Campinas, Fundação Cargill, 1988. 93p.

NEWMAN, J .E. Climate in the 1970'S. Crops Soil Mag., 22:912, 1970.

PEREIRA, H.H.G. Índices de erosividade da chuva: distribuição e relação com a preci pitação em Piracicaba-SP. Piracicaba, Escola Superior de Agricultura "Luiz de Queiroz", 1983. 70p. (Tese de Mestrado) 
ROQUE, C.G. \& CARVALHO, M.P. Distribuição da erosividade da chuva de Novo Horizonte (SP). In: CONGRESSO BRASILEIRO DE CIÊNCIA DO SOLO, 27., Brasília, 1999, Anais. Brasília, Sociedade Brasileira de Ciência do Solo, 1999. CD-ROM

ROQUE, C.G.; CARVALHO, M.P. \& PRADO, R.M. Fator erosividade da chuva dePiraju (SP): distribuição, probabilidade de ocorrência, período de retorno e correlação com o coeficiente de chuva. R. Bras. Ci. Solo, 25:147-156, 2001.

SCHWAB, G.O.; FREVERT, R.K.; DMINSTER, T.W. \& BARNES, K.K. Soil and water conservation engineering. 2.ed. New York: J ohm Wiley, 1966. 683p. (The Fergunson Foundation Agricultural Engineering Series)
SOSA, D.A. Erosividade a chuva: distribuição e correlação com a perda de solo de Pindorama, São Paulo, Brasil. Piracicaba, Escola Superior de Agricultura "Luiz de Queiroz", 1987. 105p. (Tese de Mestrado)

WISCHMEIER, W.H. A rainfall erosion index for a universal soil-loss equation. Proc. Soil Sci. Am., 20:246-249, 1959.

WISCHMEIER, W.H. \& SMITH, D.D. Predicting rainfall erosion losses from cropland east of the Rocky Mountains: guide for selection of practices for soil and water conservation. Washington, USDA, 1965. 47p. (Agriculture handbook, 282)

WISCHMEIER, W.H. \& SMITH, D.D. Predicting rainfall erosion Iosses: a guide to conservation planning. Washington, USDA, 1978. 58p. (Agriculture handbook, 537) 